\title{
Evaluation of Grain Growth and Bonding in Roll Bonded Aluminum Alloy Plates
}

\author{
E. A. Kenik, ${ }^{*}$ T. J. Huxford, ${ }^{*}$ J.R. Mayotte, ${ }^{*}$ T. S. Nedkova, ${ }^{*}$ and J. D. Sease, ${ }^{*}$
}

*Oak Ridge National Laboratory, Bldg. 4515, MS6064, Oak Ridge, TN 37831-6064

Roll bonding of metals is used to produce a variety of materials, ranging from multi-layer coinage to fuel and control plates for nuclear reactors. In the latter application, hot rolling of aluminum alloy (6061 in current study) plate must result in reproducibly bonded plates exhibiting high bond integrity and resistance to water permeability. The quality assurance specification for the control plates calls for bend testing for evaluation of interface bonding and microstructural characterization to determine the percentage of grain growth across the bond interface. The grain growth is taken as an indication of good metallurgical bonding. Optical microscope (OM) examination of etched plate cross-sections with polarized light is the established technique for microstructural evaluation. Optical microscopy indicated regions of (1) obvious grain growth across the bond line (good bonding), (2) no obvious grain growth across the bond line (no bonding) and (3) indeterminate areas. These latter areas do not change contrast when the polarization direction of the light is rotated relative to the sample. In such regions it is not possible to determine if the grains on either side of the bond line are the same or different grains. Previous reports give two numbers for the percentage of bond line exhibiting grain growth across the interface (a maximum value including both the ambiguous and "bonded" regions and a minimum including only the "bonded" region that definitely exhibited grain growth). In certifying the current production run of control plates, orientation imaging microscopy (OIM) was used to supplement optical microscopy. Three different materials prepared with the same nominal process were compared: 3 plates manufactured in 1993 (1993A-C), a pre-production plate from 2004 (2004A) and a 2004 compact dummy containing no neutron absorber material (CD). Current results were compared to previous OM evaluation of the 1993 material (1993B, C).

The OM data in Table 1 uses the same maximum/minimum convention for the current examination and presents both sets of data for comparison. There are significant differences between the CD material and the rest of the plates in the grain growth across the interface. This is true if either value is used for comparison. The combined studies show little difference between results from transverse (perpendicular to the long direction of the plate) versus longitudinal (containing the long direction) sections and the values for the current plate (2004A) match those for the earlier runs (1993A-C). For both studies, the "ambiguous" regions represent $20-50 \%$ of the bond length resulting in large error bars for the measured "bonding", especially for the CD material where the "ambiguous" bond length is $>2.5 \mathrm{x}$ greater than that of the "bonded" region. The "bonded" percentage for the CD material is less than the $20 \%$ required in the HFIR flat plate specification and correctly indicates that this material is suspect, as confirmed by ultrasonic tests and by its interfacial failure in bend tests.

OIM mapping was performed on specimens re-polished with colloidal silica. Hardness indents were used to mark the location of the bond line on etched specimens prior to re-polishing. Typical OIM maps for the 1993A and CD plates are in Figure 1. The high percentage of grain growth across the bond line for the 1993A plate (a) and low percentage for the CD plate (b) are obvious. Data for grain growth across the bond interface as determined by OIM are summarized in Table 2. OIM gives a better representation of the grain growth across the bond interface with less indeterminate regions $(\sim 4 \%)$. Consistent with the optical microscopy results, OIM indicates that the production plates 
(1993A and 2004A) have high percentages (>70\%) of grain growth across the bond interface, whereas the CD material had only $7 \%$ "bonded" length significantly below the minimum $20 \%$ required in the materials specification. OIM shows little difference in grain growth across the bond line for the transverse and longitudinal cross-sections of the 1993A plate, which agrees with the optical microscopy results presented in Table 1.

Table 1 - Percentage of grain growth across bond interface via optical microscopy

\begin{tabular}{|c|c|c|c|c|c|c|}
\hline & $\begin{array}{c}1993 \mathrm{~A} \\
\text { Trans. }\end{array}$ & $\begin{array}{c}2004 \mathrm{~A} \\
\text { Trans. }\end{array}$ & $\begin{array}{c}2004 \mathrm{~A} \\
\text { Long. }\end{array}$ & $\begin{array}{c}\text { CD } \\
\text { Long. }\end{array}$ & $\begin{array}{c}1993 \mathrm{~B} \\
\text { Trans. }\end{array}$ & $\begin{array}{c}1993 \mathrm{C} \\
\text { Long. }\end{array}$ \\
\hline $\begin{array}{c}\text { "Bonded (1)" }+ \\
\text { "Ambiguous"(3) }\end{array}$ & $80 \%$ & $85 \%$ & $90 \%$ & $32 \%$ & $76 \%$ & $79 \%$ \\
\hline "Bonded" (1) & $60 \%$ & $35 \%$ & $35 \%$ & $9 \%$ & $39 \%$ & $42 \%$ \\
\hline
\end{tabular}
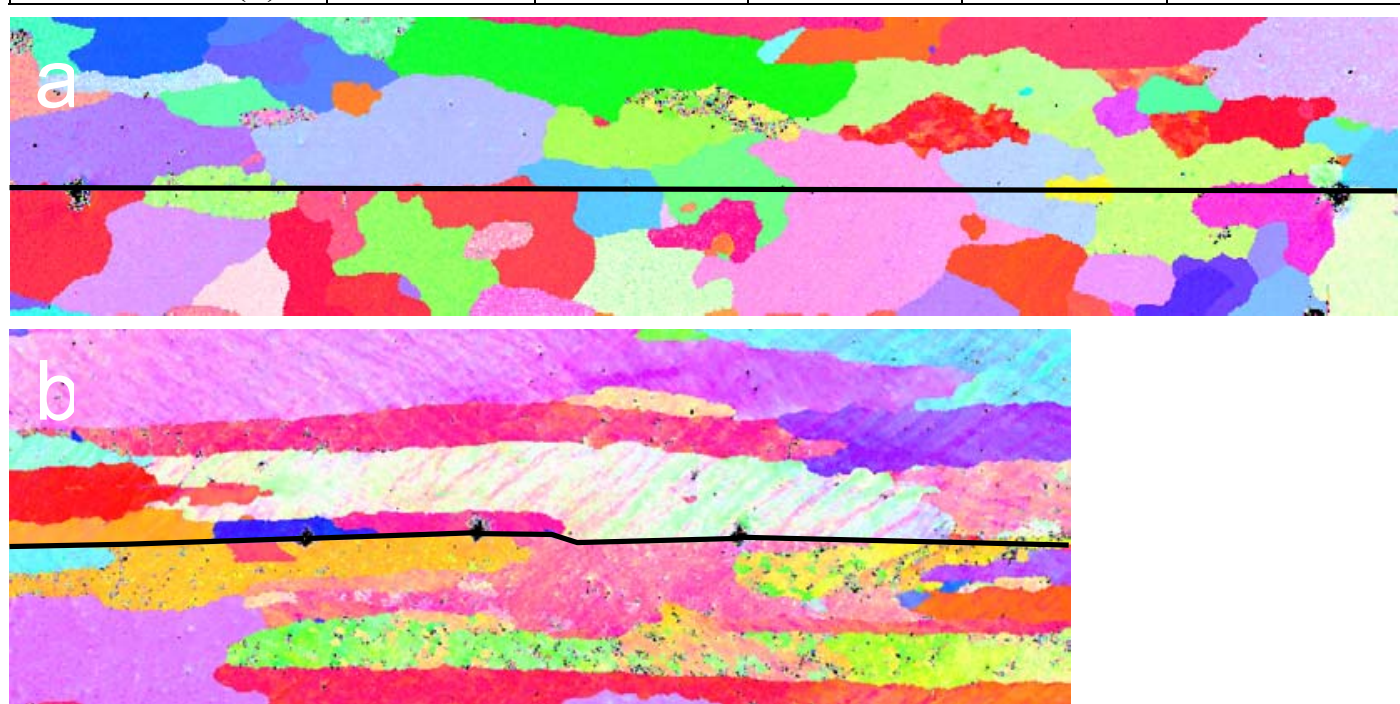

Figure 1 OIM map of grain structure of (a) 1993A (transverse section) and (b) CD (longitudinal section) plates (image widths are $\sim 6.5 \mathrm{~mm}$ and $\sim 5.0 \mathrm{~mm}$, respectively). Horizontal lines are bond lines and features on line are hardness indents used to locate bond line.

Table 2 - Percentage of grain growth across bond interface via orientation imaging microscopy

\begin{tabular}{|c|c|c|c|c|}
\hline & $\begin{array}{c}1993 \mathrm{~A} \\
\text { Trans. }\end{array}$ & $\begin{array}{c}1993 \mathrm{~A} \\
\text { Long. }\end{array}$ & $\begin{array}{c}2004 \mathrm{~A} \\
\text { Trans. }\end{array}$ & $\begin{array}{c}\text { CD } \\
\text { Long. }\end{array}$ \\
\hline "Bonded" (1) & $79 \%$ & $79 \%$ & $68 \%$ & $7 \%$ \\
\hline
\end{tabular}

Based upon these results and those of the associated bend tests, the current production run of control plates match those of the previous run and both runs exceed the required material specification for the control plates. The CD plate does not meet either the microstructural or bend test requirements. However, this failure is directly attributed to improper cleaning before roll bonding. These improper cleaning procedures were corrected after the plate CD and seven plates that have been rolled since then have passed the bend test criterion.

\section{References}

Research at Oak Ridge National Laboratory and the ORNL SHaRE User Facility was sponsored by the Division of Materials Sciences and Engineering, U.S. Department of Energy, under contract DEAC05-00OR22725 with UT-Battelle, LLC. 